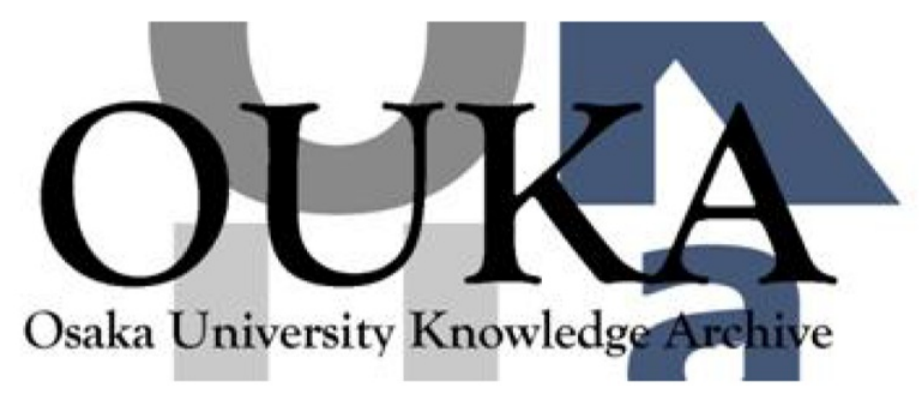

\begin{tabular}{|c|l|}
\hline Title & $\begin{array}{l}\text { Self-consistent Monte Carlo simulation of the } \\
\text { cathode fall including treatment of negative- } \\
\text { glow electrons }\end{array}$ \\
\hline Author(s) & $\begin{array}{l}\text { Dalvie, Manoj; Hamaguchi, Satoshi; Farouki, } \\
\text { Rida T. }\end{array}$ \\
\hline Citation & Physical Review A. 46(2) p. 1066-p. 1077 \\
\hline Issue Date & $1992-07$ \\
\hline oaire:version & VoR \\
\hline URL & https://hdl. handle. net/11094/78518 \\
\hline rights & $\begin{array}{l}\text { Copyright (1992) by the American Physical } \\
\text { Society. }\end{array}$ \\
\hline Note & \\
\hline
\end{tabular}

Osaka University Knowledge Archive : OUKA

https://ir. Library. osaka-u. ac. jp/

Osaka University 


\title{
Self-consistent Monte Carlo simulation of the cathode fall including treatment of negative-glow electrons
}

\author{
Manoj Dalvie, Satoshi Hamaguchi, and Rida T. Farouki \\ IBM Thomas J. Watson Research Center, P.O. Box 218, Yorktown Heights, New York 10598
}

(Received 22 October 1991; revised manuscript received 5 March 1992)

\begin{abstract}
We present results from a self-consistent Monte Carlo simulation of the cathode fall (CF) of a dc glow. The simulation consists of following electron and ion trajectories under the influence of the electric field which is calculated from Poisson's equation using the ion and electron densities. The collision cross sections are greatly simplified in order to facilitate understanding of the physical mechanisms which dominate the CF. With this aim, we explicitly sample the electron distribution function and discuss its properties. We also show that it is feasible to simulate the CF in isolation from the negative glow (NG) as long as backscattering from the NG is accounted for. Finally, we take into account the plasma (trapped) electrons in the NG, and formulate a model of the CF-NG boundary region through use of the plasmasheath equation. We find that the length of the boundary region grows with the NG density, and that there is a corresponding increase in the fraction of the ion flux which enters the CF as opposed to being created in it. For reasonable values of the NG density, we find this fraction to be as high as $25 \%$.
\end{abstract}

PACS number(s): 52.80.Hc, 52.65. $+\mathrm{z}, 52.40 . \mathrm{Hf}$

\section{INTRODUCTION}

Low-pressure, low-temperature plasmas are used extensively in the fabrication of integrated circuits. A prominent characteristic of processing plasmas is their nonequilibrium nature, which makes them difficult to analyze. A major cause of the departure from equilibrium is the presence of strong, spatially varying sheath electric fields. In dc glow discharges, much of the discharge voltage is dropped across the cathode fall (CF). Much of the nonequilibrium behavior observed in dc glow discharges therefore originates in the CF.

The CF is an important region of the glow discharge because it sustains the glow. Positive ions created in the CF by electron-impact ionization stream towards the cathode under the influence of the strong electric field in the CF. The energy gained by the ions from the electric field is transferred to the cathode and causes secondary emission of electrons from the cathode into the CF. The emitted electrons are accelerated across the CF towards the negative glow by the CF electric field. Electron acceleration in the CF leads to an electron "avalanche," as electron-impact ionization in the cathode fall creates more electrons in the $\mathbf{C F}$, which in turn are accelerated and cause more ionization [1].

The nonequilibrium nature of the CF necessitates a knowledge of the electron distribution function (EDF) in the CF. Previous theoretical studies of the cathode fall which have accounted for the nonequilibrium EDF can be categorized as either Boltzmann-equation solutions [2-8] or direct Monte Carlo simulations [9-13]. However, the two approaches are very closely related; Monte Carlo simulation can be regarded as one way to solve the Boltzmann equation for the EDF.

In addition to the work cited above, a wealth of literature also exists on studies of electron transport and avalanches that occur during electrical breakdown in gases [14-19]. The distinguishing feature of the CF studies is that the CF is characterized by a spatially varying electric field, which precludes the use of ionization and mobility data obtained from equilibrium swarm experiments.

All the Monte Carlo work cited above was performed under the assumption of a specified electric-field profile in the CF. In the majority of cases, the field was assumed to be a linearly decreasing function of distance from the cathode, down to some small field value (arbitrarily chosen) which represented the negative-glow (NG) field. The typical field configuration used is shown in Fig. 1 (the value of the NG field is exaggerated for clarity). For such a field Boeuf and Marode [9] calculated the angular and energy distribution of electrons in the CF of a helium glow discharge. They showed by comparison to a previous study [20] that angular scattering had an appreciable effect on CF properties. Ohuchi and Kubota [10] modified the linear-field assumption by using a quadratic field near the NG to account for the increase in electron density at the edge of the CF. They computed the electron-energy distribution in the CF as well as swarm parameters such as the ionization coefficient. Sato and Tagashira [11] examined the effect of the nonuniform (linear) electric field on the electron-energy distribution function (EEDF) compared to the EEDF computed from a swarm experiment. Moratz, Pitchford, and Bardsley [12] examined the applicability of swarm data (local-field approximation) to electron behavior in a linear field. Moratz [13] has also simulated the $\mathrm{CF}$ in a neon glow discharge.

The present work is based on a self-consistent calculation of the electric field based on electron as well as ion motion in the CF. In this respect, our work is closer to the work of Sommerer, Hitchon, and Lawler [2] than the 


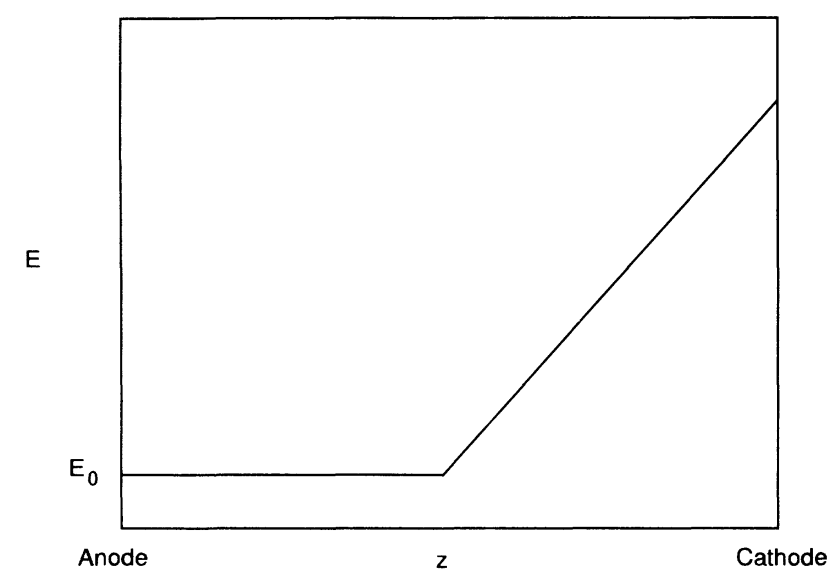

FIG. 1. "Typical" electric-field profile used in previous work.

other Monte Carlo studies. Sommerer, Hitchon, and Lawler used a weak constant NG field (as in Fig. 1), with the self-consistent calculation being done only over the nonuniform part of the field. The transition between the CF field and the weak NG field approximated the point in the discharge where the electric field (and the ion current) vanishes. This assumption was based upon experimental evidence for a field reversal near the CF-NG boundary which has been reviewed by Den Hartog, Doughty, and Lawler [21]. A field reversal in the NG has also been reported in a recent dc-discharge modeling study [22]. The scope of the self-consistent solution in [2] therefore did not include the CF-NG boundary.

In Sec. II, we present our physical model of the cathode fall. While the computer code has heen written to accept general, velocity-dependent, differential cross sections, for this study we have assumed a "model" gas with the minimum of collision physics necessary to represent the CF. We describe a few details of the simulation in Sec. III. In Sec. IV, we simulate the behavior of the model gas with the configuration used in [2], i.e., a constant, weakly positive NG field with the ion current vanishing at the field-transition point ("extended" domain). Along with the CF properties (which are in good qualitative agreement with the results in [2]), we study the effect of the value of the assumed field on the EDF in the NG. We also show that results obtained from a simulation domain that extends only as far as the CF-NG boundary (the field-transition point) are in excellent agreement with those obtained from the extended domain calculation, as long as electrons backscattered from the NG are explicitly accounted for. We develop the framework under which the CF-NG region can be analyzed in Sec. V. In Sec. VI we include the NG electrons in the simulation by replacing the fixed NG field approximation by an assumed distribution function for the NG electrons which diffuse into the CF.

\section{IDEALIZED CF MODEL}

We present below a model of the CF by which we hope to capture much of the nonequilibrium physics of the CF.
We consider three different species in the CF: neutral atoms of the background gas, singly ionized atoms of the same gas, and free electrons. Electrons are emitted through secondary processes on the cathode surface $(z=d)$. Previous studies have assumed various forms of the distribution of emitted electrons and, furthermore, have shown that the effect of the assumed form does not propagate very far into the CF $[9,21]$. We use a Maxwellian electron-energy distribution with a standard deviation corresponding to an energy of $2 \mathrm{eV}$. The angular distribution of the emitted electrons is assumed to be isotropic within a hemisphere. Emitted electrons are accelerated by the CF field towards the discharge. As they traverse the CF, they suffer collisions with neutral gas atoms, elastic as well as inelastic (excitation and ionization). Ionization in the $\mathrm{CF}$ creates an electron avalanche as newly created electrons are themselves accelerated by the CF field and undergo ionization collisions. Ions produced by ionization on the cathode side of the fieldreversal point stream towards the cathode in the CF field. (The field-reversal point is denoted by $z=-x_{0}$, and the field value is equal to a threshold value, $E_{0}$, at $z=0$.) Ions also collide with neutral gas atoms, resulting in momentum transfer to the neutral atom in either an elastic or a charge-exchange collision. Energy gained by the ion from the CF field is transferred to the cathode, resulting occasionally in the emission of an electron. The discharge is therefore self-sustaining. The electric field is created by the space charge in the CF.

This model is considered to be idealized for reasons which include the following. Metastable species and their effects are not considered; ionization is assumed to occur only from electron impact on ground-state neutral atoms. Collision processes have been greatly simplified as discussed below.

Given the nonequilibrium character of the $\mathrm{CF}$, a discussion of the CF should be based upon the Boltzmann equation. Before commencing, we introduce the definition of the distribution function (one each for ions and electrons):

$$
d n^{e}=f^{e}(\mathbf{v}, z) d^{3} \mathbf{v},
$$

where the superscript refers to electrons, $n^{e}$ is the electron number density at position $z, f^{e}(\mathbf{v}, z)$ is the distribution function, $\mathbf{v}$ is the electron velocity, and $z$ is defined on a coordinate axis parallel to the electric field. The ion distribution $f^{i}$ has an analogous definition. The geometry is assumed to be plane parallel, implying an axisymmetric distribution function which we write as

$$
f^{e}(\mathbf{v}, z)=f^{e}\left(v_{z}, v_{1}, z\right),
$$

where $v_{z}$ and $v_{\perp}$ are the axial and perpendicular components of the velocity vector.

We are interested in the steady-state behavior of the $\mathrm{CF}$ for which we may begin with the time-independent Boltzmann equation, shown below for electrons:

$$
\mathbf{v} \cdot \nabla_{x} f^{e}+\mathbf{a}^{e} \cdot \nabla_{\mathbf{v}} f^{e}=\left(\frac{\delta f^{e}}{\delta t}\right)_{\text {coll }},
$$


where $\mathbf{a}^{e}$ is the acceleration of electrons due to the CF field. Since the CF is assumed to be axisymmetric, we can reduce Eq. (3) to

$$
v_{z} \frac{\partial f^{e}}{\partial z}-\frac{q E}{m} \frac{\partial f^{e}}{\partial v_{z}}=\left[\frac{\delta f^{e}}{\delta t}\right)_{\text {coll }}
$$

An analogous equation for the ion distribution function (with a positive acceleration term) is coupled to Eq. (4). The coupling between the ions and the electrons occurs through two mechanisms. One is through Poisson's equation for the electric field:

$$
\frac{d E}{d z}=\frac{q}{\epsilon_{0}}\left(n^{i}-n^{e}\right),
$$

where the electron and ion densities are obtained by integrating Eq. (1) and its analog. The second coupling occurs through the ionization part of the collision integral, written above as $\left(\delta f^{e} / \delta t\right)_{\text {coll }}$ for the electrons. Collisions are discussed below.

\section{A. Collision processes}

A major source of difficulty in solving the coupled system formed by Eqs. (4), the analogous equation for the ions, and Eq. (5) is the collision integral terms in the electron and ion equations. While it is possible to account for elastic as well as inelastic collisions in the collision integral $[19,23]$, evaluation of the collision integral requires a priori knowledge of the distribution function. Since we use the Monte Carlo simulation to obtain the distribution function, the collision processes are described in the context of the Monte Carlo simulation.

Two types of ion-atom and three types of electronatom collisions are included in the model. Interactions between charged particles besides the effect of the spacecharge field are not included. The model gas is "argonlike"; the mass of the neutral and ionic species is $40 \mathrm{amu}$. However, while the numbers for the various collision cross sections have been chosen to reflect cross sections measured in Ar, the collision physics have been greatly simplified.

Electron-atom collisions fall under elastic, excitation, and ionization categories. Figure 2 shows the cross sec-

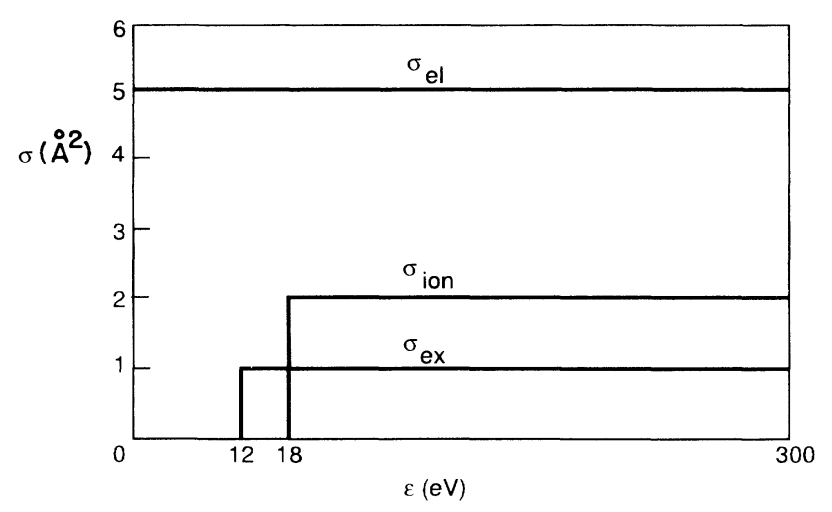

FIG. 2. Cross sections for electron-atom collisions. tions used for the collisions. Energy dependences of the cross sections have been neglected, except for the threshold behavior of the inelastic processes (12 and $18 \mathrm{eV}$ for excitation and ionization, respectively). Furthermore, angular dependences have also been ignored in favor of an isotropic scattering model for all collisions. In ionizing collisions, all allowed energy distributions between the ionizing electron and the progeny have equal probability. The values for the cross sections in Fig. 2 are based upon [24-26]. We use the null collision technique to account for the ionization and excitation threshold.

Collisions between ions and neutral atoms are either charge-transfer collisions or elastic collisions. Charge exchange is modeled as an "identity switch" between the ion and neutral atom with no further interaction, resulting in a new ion with an initial velocity corresponding to a neutral atom. The newly created "hot" neutral atom is not followed, and is assumed not to alter the neutralatom distribution significantly. While this is a possible source of error [21], we regard it as a second-order effect for the purpose of this study. Elastic collisions are modeled after hard-sphere interactions. The cross sections for the ion-atom collisions are $30 \AA^{2}$ (charge exchange ) and $10 \AA^{2}$ (elastic). The numbers are based upon experimental measurements of $\mathrm{Ar}^{+}-\mathrm{Ar}$ collisions [27], but variations with energy and scattering angle have been neglected. The neutral gas is assumed to follow a Maxwellian distribution with a temperature of $350 \mathrm{~K}$.

While the collision models adopted here are crude, we show that much of the CF behavior seen in previous studies is reproduced by this model. Furthermore, the simplicity of the collision processes aids our understanding of this behavior.

\section{B. Flux boundary conditions}

In previous studies of electron avalanches in the CF, boundaries of the simulation have been physical electrodes, typically regarded as being absorbing $[2,9,10]$. Electron reflection from solid surfaces is ignored here as well. Electrons are emitted at the cathode through secondary processes initiated by ion, neutral-atom, and/or photon impingement. In a fixed-field simulation of electron transport, it is possible to specify the electron flux at the cathode (or at any other place) as a sufficient boundary condition. In self-consistent simulations, however, it is necessary to use a relationship between the electron and ion currents. We use the concept of a current balance at the cathode, studied by Doughty, Den Hartog, and Lawler [28]. The ratio of the electron current to the ion current at the cathode is defined as $\gamma$

$$
\gamma=\frac{j_{e}(d)}{j_{i}(d)},
$$

where $j_{e}$ and $j_{i}$ are the electron and ion currents, and $d$ is the position of the cathode. It is important to note that $\gamma$ is not the secondary yield per impinging ion; the condition is on the flux of particles. This condition has also 
been used in [2].

At the field-reversal point in the NG $\left(z=-x_{0}\right)$, we assume that the current is carried exclusively by electrons. This assumption is derived from Sommerer, Hitchon, and Lawler [2] and Den Hartog, Doughty, and Lawler [21]. It follows that the net ion flux across this boundary is zero. The ion flux is primarily due to field-induced drift [22], and therefore vanishes at the field-reversal point. An alternative expression for $\gamma$ in terms of electroncurrent amplification is

$$
j=j_{e}(d)\left(1+\frac{1}{\gamma}\right),
$$

where $j$ is the total discharge current. In the extended domain simulations, the field-reversal point is approximated by the transition from the CF field to the fixed NG field $\left(x_{0}=0\right)$.

\section{MONTE CARLO SIMULATION}

The Monte Carlo simulation consists of following trajectories of individual particles. The scope of the simulation includes ion trajectories between their creation in the $\mathrm{CF}$ from electron-impact ionization and their ultimate absorption by the cathode, as well as the electron avalanche resulting from the secondary emission of electrons from the cathode. This work is based largely on an earlier Monte Carlo simulation of ion transport [29]. The reader may refer to [29] for more details of the simulation.

Figure 3 shows a flow chart of the overall simulation. Input data consist of initial guesses for the electric field, the electron-density profile, and the ionization profile in the CF (usually results from a previous run). Ion trajec-

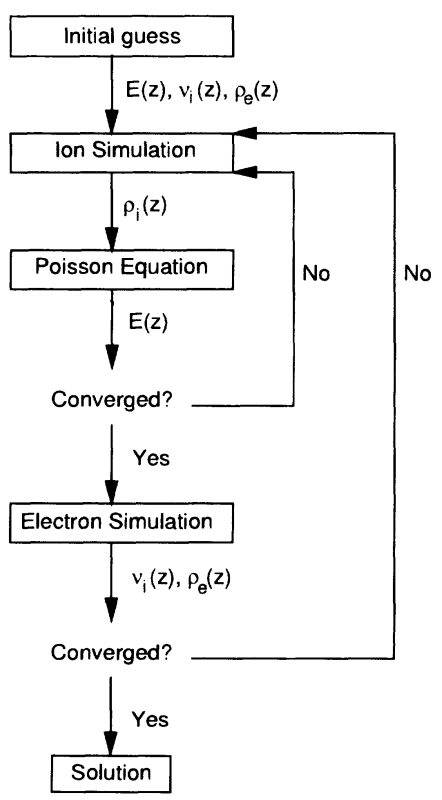

FIG. 3. Algorithm for self-consistent Monte Carlo calculation. tories are followed from their starting position until they cross the plane of the cathode. From the averaged motion of a specified number of test particles, the iondensity profile in the CF is computed. Through Poisson's equation, Eq. (5), a new electric field is calculated. Poisson's equation is integrated using a straightforward finite-difference scheme; particle densities are not smoothed or filtered prior to the integration. The $L_{1}$ norm of the difference between the new and old field profiles is computed and compared to a specified tolerance value. If the difference is too large, a new set of ion trajectories is generated. This procedure is repeated until the electric-field profile converges.

The converged electric field is then used to generate the electron avalanche. A specified number of test particles representing electrons are started from the cathode. Trajectories of these as well as of electrons generated in the CF are averaged to obtain the electron-density and ionization profiles in the CF. The newly generated ionization profile is compared to the previous ionization profile to test for convergence. If the error is larger than the specified tolerance, the new electron-density and ionization profiles are used in the ion simulation to generate a new electric field and the process is repeated. The use of the ionization profile as the error criterion allows us to test the convergence of a specific part of the electron distribution (the high-energy tail) as a function of position in the CF. The price we pay (over testing, for instance, a macroscopic average such as the drift velocity) is computer CPU time since we need to run a large number of particles to populate the high-energy tail of the distribution to obtain reasonable statistics.

For a given set of collision cross sections, there are four parameters which characterize this problem. They are the discharge current $(j)$, the CF thickness $(d)$, the CF potential $(V)$, and the cathode current balance $[\gamma$ from Eq. (6)]. In a self-consistent solution, two of these parameters can be set independently. Sommerer, Hitchon, and Lawler [2] used $j$ and $\gamma$ as the independent variables and calculated $V$ and $d$ as dependent variables. We find it more convenient numerically to fix $j$ and $d$ and calculate $V$ and $\gamma$.

\section{Sampling of the electron distribution function}

We sample the electron distribution function more extensively than the ion distribution. The discussion in this section, however, applies to ions as well as to electrons.

The EDF and its moments are sampled at constant time intervals before each integration step. The spatial extent of the domain is discretized into $N_{z}$ "space bins" of equal size such that each bin is smaller than the mean free path $\left(\Delta z=d / N_{z}<\lambda\right)$. The moments of the distribution function (e.g., the drift velocity $\left\langle v^{e}\right\rangle$ ) are calculated by first adding the sample of the relevant quantity (e.g., the velocity component $v_{z}$ parallel to the field) to a cumulative sum, and then dividing by the number of samples in the cumulative sum [29]. In terms of the distribution function, this amounts to calculating the drift velocity as 


$$
\left\langle v^{e}\right\rangle=\frac{\int f^{e} v_{z} d^{3} v}{\int f^{e} d^{3} v},
$$

where $f^{e}$ is the EDF. By inference, the sample count (the number of samples taken) is related to the electron density [the denominator in Eq. (8)]. Since trajectories are sampled at constant time intervals, this is consistent with previous sampling techniques employed [16]. It is also intuitive, since a slow electron which contributes more to the density is sampled more often than a fast electron. Other moments of the distribution function calculated the same way as Eq. (8) are the variance of the axial and perpendicular velocity components $\left\langle\left(v_{z}-\left\langle v^{e}\right\rangle\right)^{2}\right\rangle$ and $\left\langle\left(v_{\perp}\right)^{2}\right\rangle$, used to assess "temperatures" and pressure gradients in the parallel and perpendicular directions.

As discussed in [29], the Monte Carlo simulations must be interpreted as modeling events which occur in a fixed time interval. By this interpretation, the number of electrons which cross a hypothetical plane are representative of the $f u x$ at that plane. Therefore the flux at a point is calculated by counting the number of times each electron crosses that point and normalizing the count as

$$
j_{e}(z)=j \frac{C_{e}(z)}{C_{e}\left(-x_{0}\right)},
$$

where $j_{e}(z)$ is the electron current (flux) and $C_{e}(z)$ is the net particle-crossing count for a plane at position $z$. The discharge current $j$ is carried exclusively by electrons at the field-reversal point $\left(z=-x_{0}\right)$. In the extended domain simulation, $x_{0}=0$. This method of counting the flux is based upon the same line of argument used in [9].

Equations (8) and (9) are two different methods of arriving at the same information. For instance, the numerator in Eq. (8) is the definition of the flux and we obtain that directly as an intermediate step in the driftvelocity calculation. We have confirmed that the two methods give the same results. Our reason for choosing Eq. (9) over Eq. (8) for the flux calculation is that the agreement between the ion flux and the electron flux at each position (they must add to a constant value) shows less statistical variation by Eq. (9).

On the other hand, the method of Eq. (8) is preferable for the calculation of the distribution function. In order to calculate the distribution function through the flux [Eq. (9)] it is necessary to estimate the time that each electron spends in each spatial bin. For curvilinear trajectories in arbitrary electric fields, this calculation is not possible analytically. In a previous application of this method, Boeuf and Marode [9] used a first-order approximation for $\Delta t$ by assuming that acceleration of the electron over the extent of a single spatial bin was negligible. The method of Eq. (8), outlined below, is free of this assumption. However, the trajectory integration scheme used here (which allows us to use either method) is more CPU intensive than that used in [9].

As stated above, the denominator in Eq. (8) (the density, by definition) is directly proportional to the total number of samples taken (as a function of position) from all particles. The sample count is made without regard to the velocity or energy of the particle. If the count is "binned" by velocity (energy), this yields the velocity (energy) distribution of the electrons. The binning method is very simple; every time a particle is sampled, the appropriate velocity (energy) bin for that sample is calculated based upon the current velocity (energy) of the particle. That particular velocity (energy) bin is incremented by one. It is possible by this method to calculate the full, exact distribution function $f^{e}\left(v_{z}, v_{\perp}, z\right)$, but the statistical requirements prove too demanding of CPU time. Therefore we restrict ourselves to computing the following distributions:

$$
\begin{aligned}
& F^{e}\left(v_{z}, z\right)=\int_{0}^{\infty} f^{e} v_{\perp} d v_{\perp}, \\
& G^{e}\left(v_{\perp}, z\right)=v_{\perp} \int_{-\infty}^{\infty} f^{e} d v_{z}, \\
& H^{e}(\varepsilon, z)=\int_{0}^{\pi} f^{e^{\prime}}(\varepsilon, \theta, z) \sin \theta d \theta,
\end{aligned}
$$

where $f^{e}$ is related to $f^{e^{\prime}}$ in Eq. (12) through a (phasespace) coordinate transformation

$$
f^{e}\left(v_{z}, v_{\perp}, z\right)=\left(\frac{2}{m_{e}}\right)^{-3 / 2} \varepsilon^{-1 / 2} f^{e^{\prime}}(\varepsilon, \theta, z)
$$

and $\varepsilon, \theta$ have the conventional definitions

$$
\begin{aligned}
& v_{z}=\left(\frac{2 \varepsilon}{m_{e}}\right)^{-1 / 2} \cos \theta, \\
& v_{\perp}=\left(\frac{2 \varepsilon}{m_{e}}\right)^{-1 / 2} \sin \theta .
\end{aligned}
$$

The distributions given by Eqs. (10)-(12) are normalized through the electron density $n^{e}$ as

$n^{e}(z)=\frac{j_{e}(z)}{q\left\langle v^{e}\right\rangle}=\int_{-\infty}^{\infty} F^{e} d v_{z}=\int_{0}^{\infty} G^{e} d v_{\perp}=\int_{0}^{\infty} H^{e} d \varepsilon$,

where $j_{e}$ is given by Eq. (9) and $\left\langle v^{e}\right\rangle$ is from Eq. (8).

\section{EXTENDED-DOMAIN SIMULATION}

In order to compare the calculation of the electron properties with previous work $[2,9,22]$, we present some properties of the EDF in Fig. 4, along with the (selfconsistent) electric field by which the results are generated. As indicated in Fig. 4, the domain for this calculation is extended to the anode $(z=-d)$. The electrodes are equidistant from the CF-NG boundary $(z=0)$. A weak NG field $\left(E_{0}=10 \mathrm{~V} / \mathrm{cm}\right)$ pointing towards the cathode is assumed, following [2] and [9], and the self-consistent field calculation is done only for $z>0$. The EDF is normalized everywhere by equating the electron current at $z=0$ to the specified discharge current [Eq. (9)].

The parameters used to generate the results in Fig. 4 are $d=4 \mathrm{~mm}$ and $j=2 \mathrm{~mA} / \mathrm{cm}^{2}$. The simulation yields the CF voltage $V=305 \mathrm{~V}$ and electron- to ion-current ratio at the cathode $\gamma=0.045$.

The electric field shown in Fig. 4 is from the selfconsistent motion of ions and electrons in the CF $(z>0)$. Ion behavior is discussed below. The field shows nearly 


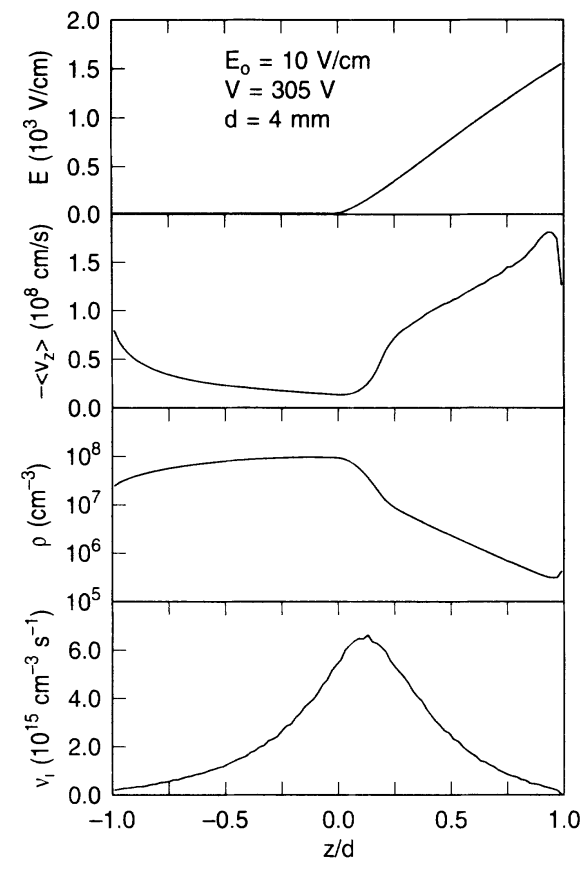

FIG. 4. EDF properties from extended-domain simulation.

linear behavior in the CF, consistent with the known behavior of the CF [21]. The drift-velocity and density profiles may be compared directly with results from [2,9]. It is apparent through such comparison that the behavior is qualitatively similar to that reported earlier, the crude collision model notwithstanding. We discern three distinct regions in the CF. Very close to the cathode, the drift velocity increases (density drops) as the low-energy emitted electrons are accelerated away from the cathode. In the bulk of the CF, the velocity is determined by the local field value in a balance between the momentum gained from the field and the randomizing effect of isotropic collisions. As the field decreases towards the NG, the drift velocity therefore drops and the density increases.

The third region is marked by the sharp decrease in the drift velocity which occurs at $z / d \approx 0.2$. This region of the CF is affected by the NG; the observed drop in the drift velocity is due to electrons being backscattered from the low-field region. This feature, though visible, is not so pronounced in the model results of [2], presumably because of the anisotropic scattering functions and the greater number of scattering processes included in [2]. The backscatter from the NG may be construed as a pressure gradient which the electrons must overcome as they "flow" from the cathode to the anode, and which begins to play a large retarding role in the momentum balance at roughly $z / d \approx 0.2[30]$.

The drift velocity in Fig. 4 rises smoothly from the CF-NG boundary towards the absorbing anode due to the decreasing number of backscattered electrons. It is interesting to note that with the isotropic scattering model used here, the effect of the absorbing anode is felt at least as far away as the CF-NG boundary. This effect is not so strong in studies using anisotropic scattering $[2,9]$ and the anode is completely decoupled from the system in the limit of pure forward scattering [20].

The electron density, calculated according to Eq. (16), varies with position due to two mechanisms. The electron flux increases from the cathode towards the anode, causing the density to increase proportionately. In the $\mathrm{CF}$, the decrease in the drift velocity enhances this effect; from the correspondence of the velocity and density profiles, it is clear that this is not an insignificant contribution. However, the density is plotted on a logarithmic scale in Fig. 4, and the effect of the flux amplification cannot be discounted. In the NG, the flux amplification and the drift-velocity gradient work against each other to produce a density profile more constant than the driftvelocity profile in the same region.

The ionization rate $v_{i}$ shown in Fig. 4 is indicative of the growth of the electron flux $\left(q v_{i}=-d j_{e} / d z\right)$. The profile is qualitatively similar to those shown in [22], peaking close to the CF-NG boundary. Since the ionization rate is dependent upon the average electron energy (which peaks inside the CF) and the electron density (maximum in the NG), it is not surprising that the position of the maximum rate is near the CF-NG boundary.

It is of interest to examine the behavior of the highenergy component of the EDF as a function of position. In Fig. 5 we show the absolute densities of electrons above the ionization and excitation potentials, as well as the fraction of the total density residing above the inelastic potentials. The logarithmic scale in Fig. 5(a) notwithstanding, the ionization rate profile in Fig. 4 closely follows the density of electrons above the ionization threshold. Comparison between the two curves in Fig. 5 shows immediately that the drop in the fraction [Fig. 5(b)] at $z / d \approx 0.2$ to due to the rise in the total density, since the absolute densities in Fig. 5(a) show qualitatively different behavior. As in the drift-velocity discussion above, this is the effect of the low-field region of the discharge on the density of the low-energy electrons (see Fig. 7 and discussion below).

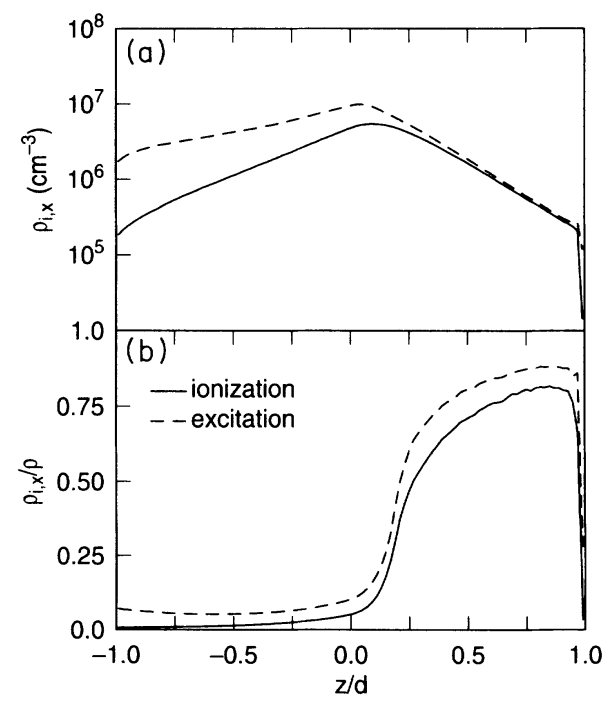

FIG. 5. (a) Absolute and (b) relative densities of electrons above ionization and excitation potentials. 


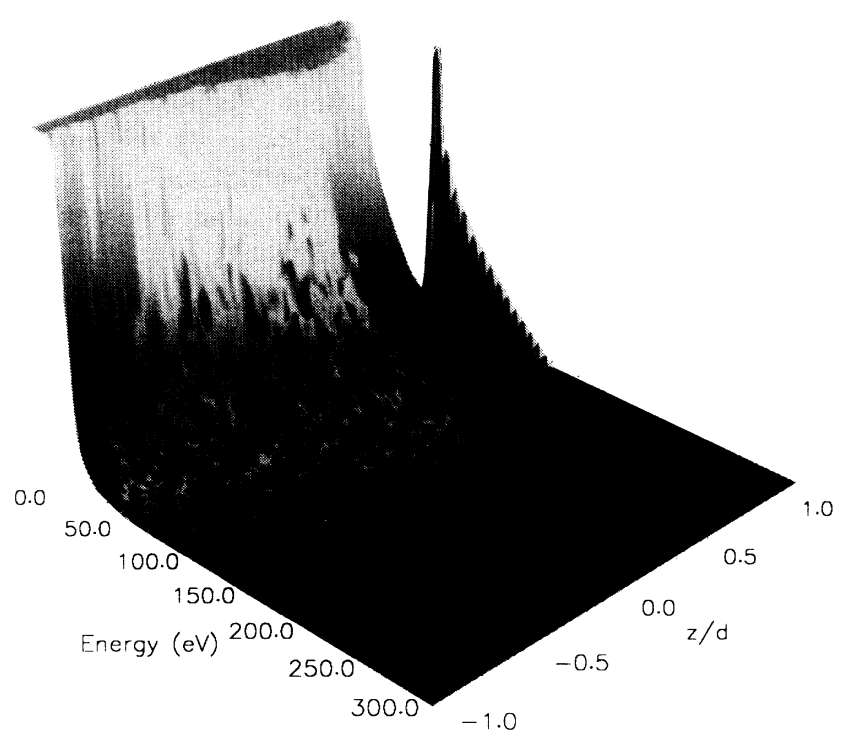

FIG. 6. EEDF as a function of position.

Before proceeding to the results of the ion calculations, we show the EEDF [from Eq. (12)] as a function of position in the CF in Fig. 6. The vertical scale of Fig. 6 has been expanded to show the structure adjacent to the cathode. Those electrons which have not undergone any inelastic collisions are clearly visible as the curved ridge near the cathode. Electrons which have collided inelastically and secondary electrons from ionization add to the growth of the low-energy electron density away from the cathode.

Figure 7 shows the EEDF at the edge of the CF $(z=0)$ for three different values of the weak NG field. The energy scale is truncated at $50 \mathrm{eV}$ to show the structure at low energies. The value of the NG field (we used 10, 1, and $0.1 \mathrm{~V} / \mathrm{cm}$ ) affects only the low-energy part of the distribution; the high-energy tail (which extends, with slight positive curvature on the logarithmic plot, to the sheath potential of $300 \mathrm{eV}$ ) is unaffected. Therefore ionization

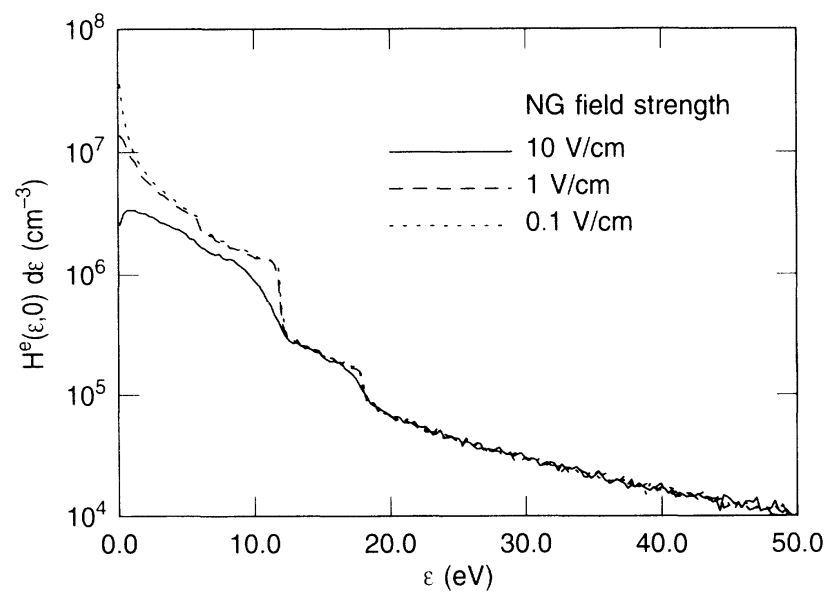

FIG. 7. EEDF at CF-NG boundary using different assumed NG field strengths. and excitation rates are independent of the NG field. At low energy, the ionization and excitation energy loss can be seen clearly in Fig. 7. The effect of the inelastic collisions is to remove electrons from energies higher than the respective potentials and add them to the distribution at lower energy: hence the sharp steps visible in Fig. 7 at $12 \mathrm{eV}$ (excitation threshold) and $18 \mathrm{eV}$ (ionization threshold). At lower values of the NG field, the density of the low-energy electrons (and the total electron density) rises, as expected.

Turning our attention now to the ion calculation, we present the drift-velocity, the density, and the flux profile in Fig. 8. The electron density (from Fig. 4) and the electron flux are also shown. As noted before, the ion transport calculation is performed only in the CF $(z>0)$ since the weak NG field actually approximates a reversed field in the NG and ions produced in the NG drift towards the anode. The ion drift velocity is seen to increase as the field strength increases. Consistent with the near-linear electric-field profile in Fig. 4, the variation of the ion density in the CF is small compared to the electrons. However, it is apparent that the maximum ion density is found inside the CF; the density of ions decreases towards the NG. Such a profile seems nonphysical since the ion density is higher in the NG than in the CF [22]. While not obvious in the figure, the ion density at the edge of the CF is only $40 \%$ of the maximum ion density. Since the electric-field calculation is dominated by the iondensity profile $\left(n^{i}-n^{e}\right.$ from Fig. 8 is quite large), this could affect the electric field significantly.

Lawler [31] has shown by analyzing the Boltzmann equation for the ion drift that the ion velocity inside the CF is close to the equilibrium drift velocity, except in the "equilibration" region close to the NG. This conclusion has been utilized in two previous studies to approximate ion motion in the CF $[2,22]$. Through swarm simulations in constant electric fields, we have calculated the ion mobilities at different field strengths in the range of the

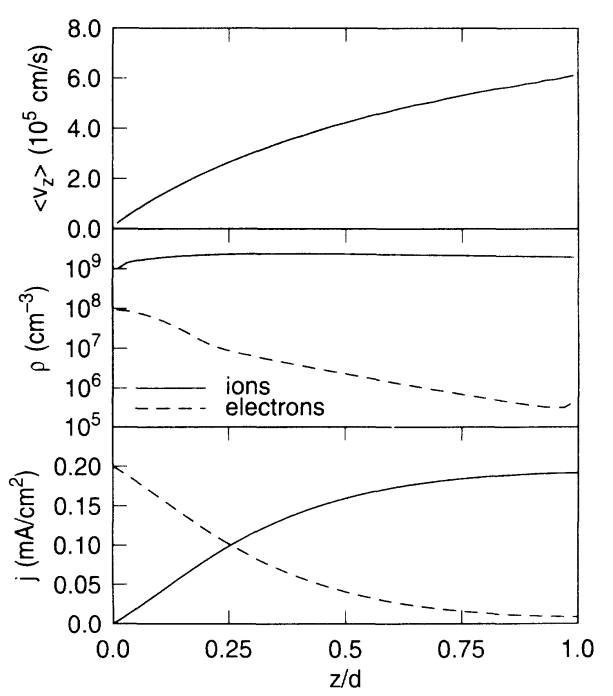

FIG. 8. Ion and electron behavior from extended-domain simulation. 


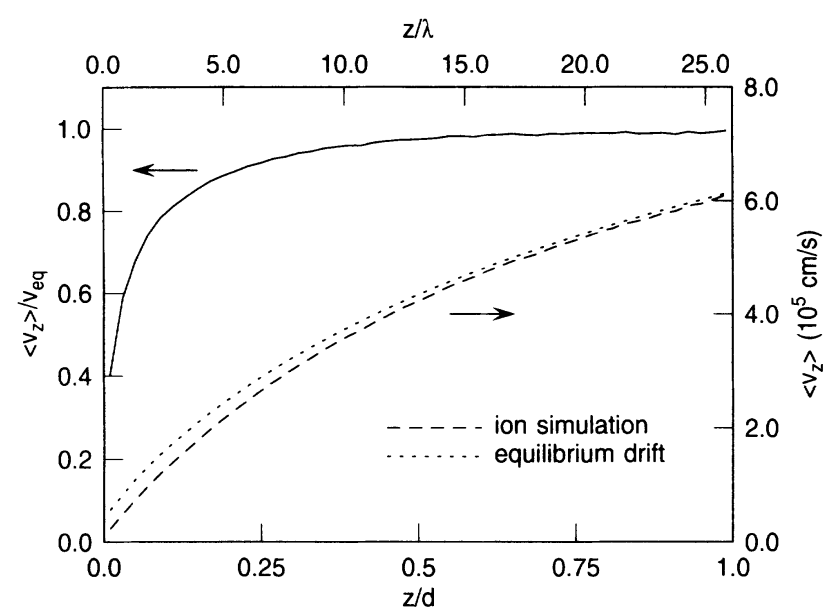

FIG. 9. Ion drift velocity from simulation and equilibrium calculation.

CF electric field. The mobilities thus found conform well to the functional form suggested by Frost [32]

$$
\mu=\frac{\mu_{0}}{\sqrt{1+a E / n_{g}}},
$$

with the mobility $\mu$ approaching $\mu_{0}=7350 \mathrm{~cm}^{2} / \mathrm{V} \mathrm{s}$ at low field due to the gas temperature and showing the inverse square-root dependence on $E / n_{g}$ at high field $\left(a=0.036 \mathrm{Td}^{-1}, 1 \mathrm{Td}=10^{-17} \mathrm{~V} \mathrm{~cm}^{2}\right)$. Figure 9 shows the ratio of the drift velocity from the simulation to the equilibrium drift. The two drift velocities are also shown. The results are consistent with Lawler's conclusion that the equilibration distance (defined as the distance over which the ions achieve $90 \%$ of the equilibrium drift) is on the order of 5 mean free paths. While Lawler considered only charge-exchange collisions, we observe qualitatively similar behavior from ions which undergo hard-sphere elastic collisions with neutral species of the same mass.

We retain the ion-transport simulations for two reasons. First, we are interested in the CF-NG boundary, the equilibration region, where the fractional discrepancy between the equilibrium and true drift velocities is large. Note that use of the higher equilibrium velocity in this region drives the ion density lower at the CF-NG boundary, worsening the discrepancy in the ion density discussed above. Second, the ion calculation is computationally inexpensive compared to the electron calculation.

The flux profiles in Fig. 8 reflect the ionization profile in Fig. $4\left(d j_{i} / d z=-d j_{e} / d z=q v_{i}\right.$ by continuity). The total discharge current is $0.2 \mathrm{~mA} / \mathrm{cm}^{2}$, and it is assumed in the model that the current is carried totally by electrons at the edge of the CF $(z=0)$. The ion current is therefore assumed to vanish at this point.

\section{Effect of backscattering from NG}

In Fig. 10, we show the axial velocity distribution of the electrons $\left[F^{e}\left(v_{z}, 0\right)\right.$ from Eq. (10)] calculated from the extended simulation at the CF-NG boundary. On the

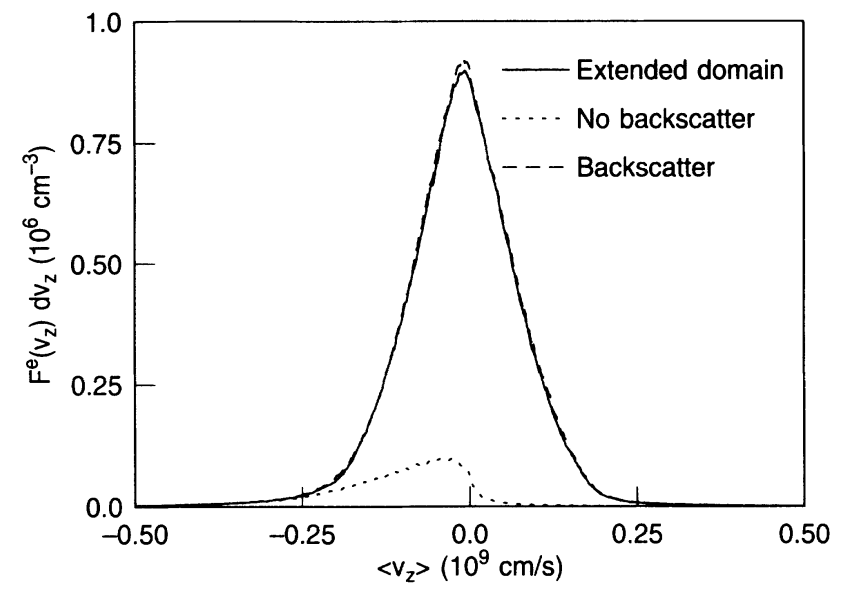

FIG. 10. Axial velocity distributions obtained from extended- and shortened-domain calculations.

same plot, we show the axial velocity distribution obtained by simulating only the CF. The lack of backscattering from the NG (the CF-NG boundary is treated as a virtual anode) creates a one-sided distribution and reduces the density as well. Also the value of $\gamma$ rises from 0.045 to 0.055 , indicating that the backscattered electrons contribute to ionization as well as to the density. To confirm this mechanism, we introduce particles at the CF-NG boundary with axial velocities corresponding to the $v_{z}>0$ (reentering the CF) portion of $F^{e}\left(v_{z}, 0\right)$. Perpendicular velocities of the backward-going particles are distributed according to the perpendicular velocity distribution $G^{e}\left(v_{1}, 0\right)$ [Eq. (11)] from the extended solution. The resulting axial velocity distribution, shown in Fig. 10 , compares very favorably with the distribution from the extended domain simulation, as does that the perpendicular velocity distribution. The value of $\gamma$ also returns to its extended domain simulation value of 0.045 . It should be noted that the application of Eqs. (10) and (11) to determine the velocity distributions of the backwardgoing particles assumes that the distribution function $f^{e}\left(v_{z}, v_{\perp}\right)$ is separable. From Fig. 10, the error due to the assumption appears to be small.

\section{TREATMENT OF NEGATIVE GLOW}

In the results shown in Fig. 4 as well as in [2], the rise in the electron density towards the NG is not sufficient to account for the observed NG density of $10^{11} \mathrm{~cm}^{-3}[21]$. Also, the ion-density profile peaks inside the CF (Fig. 8), contrary to experimental observations [21] and full discharge simulation results [22]. This reduces the overall accuracy of the self-consistent electric-field calculation. As pointed out in [2], the rigorous solution to this problem would involve an electrode-to-electrode simulation, including the reversed field, which would be computationally expensive. Therefore we use a model of the CF-NG region to find appropriate boundary conditions for the Monte Carlo simulation.

Experimental measurements of the EEDF in the NG indicate that electrons may be classified into two groups, 
trapped (plasma) electrons and a high-energy group referred to here as a beam [33,34]. (In [33], "beam" electrons refer to those electrons which pass through the CF without a single inelastic collision; under our simulation conditions, very few electrons escape inelastic collisions in the CF. We use "beam" to refer to the high-energy tail of the distribution shown in Fig. 7, even though the electrons in question are not necessarily directed towards the anode.) Figure 7 shows that the beam electrons are satisfactorily described in the NG by the simulation of the extended domain using a constant, weakly positive NG field. The trapped plasma electrons are described phenomenologically as Maxwellian, with a parametrized density and an assumed temperature $(0.25 \mathrm{eV}$ [21]).

Since the temperature of the plasma electrons is low compared to the ionization and excitation potential, they only affect the system through the electron density. For this purpose, it is convenient to express the density of the plasma group directly as a function of position instead of actually injecting particles in the Monte Carlo simulation. Therefore the density of plasma electrons is given analytically by

$$
n_{p}^{e}(z)=n_{p}^{e}(0) e^{V(z) / T_{e}},
$$

where $V(z)$ is the potential in the CF ( $V=0$ at the fieldreversal point) and $T_{e}$ is the electron temperature in $\mathrm{eV}$ (assumed $0.25 \mathrm{eV}$ ). The density at the field-reversal point is represented symbolically by $n_{p}^{e}(0)$. The total electron density is given by the sum of the plasma component [Eq. (18)] and the beam component (from the simulation) of the EDF.

The CF-NG boundary is most conveniently analyzed in terms of the plasma-sheath equation, which was first formulated by Tonks and Langmuir [35] and has subsequently received attention from a number of researchers [36-38]. Although the equation is usually written for a symmetric discharge, its use here is consistent with the assumption that there is no net ion transport across the boundary where the field vanishes. It is convenient to use the potential at the field-reversal point as the reference (zero) potential. Ignoring the low density of beam electrons, the plasma-sheath equation takes the form

$$
-\frac{\epsilon_{0}}{q} \frac{d^{2} V}{d z^{2}}=\int f^{i}(\mathbf{v}, z) d \mathbf{v}-n_{p}^{e}(0) e^{V(z) / T_{e}}
$$

where $f^{i}$ is the ion distribution function. In order to proceed with the analysis, it is necessary to specify a form for $f^{i}$. Previous applications of this equation have been mostly restricted to a collisionless plasma $[35,36]$ or to a highly collisional plasma [35]. However, ions under these conditions are not collisionless and the results in Fig. 9 confirm Lawler's finding that ions in the CF-NG region are not in equilibrium with the field [31].

For preliminary analysis, we consider the ionization rate $v_{i}$ due to the beam component of the EDF to be uniform. For the case of only charge-exchange collisions between ions and cold neutral atoms it is possible to relate $f^{i}$ to the potential $V$ as follows [39]:

$$
f^{i} d v=\left(\frac{m_{i}}{2 q}\right)^{1 / 2} \frac{v_{i} e^{-(z-\xi) / \lambda_{c}}}{\sqrt{V(\xi)-V(z)}}\left(1+\frac{\xi}{\lambda_{c}}\right) d \xi,
$$

where $m_{i}$ is the ion mass, $\lambda_{c}$ is the mean free path for charge exchange, $z$ is independent coordinate variable, and $d \xi$ is the region around $\xi$ where the ions arriving at $z$ either (i) underwent their last charge-exchange collision or (ii) were created before arriving at $z$ before colliding. Substituting Eq. (20) into (19) we can write in nondimensional form:

$\frac{\lambda_{D}^{2}}{L^{2}} \frac{d^{2} \eta}{d s^{2}}=\int_{0}^{s} \frac{e^{-(s-\sigma) / l}}{\sqrt{\eta(s)-\eta(\sigma)}}\left(1+\frac{\sigma}{l}\right) d \sigma-e^{-\eta}$.

Here, $\lambda_{D}=\left[\epsilon_{0} T_{e} / q n_{p}^{e}(0)\right]^{1 / 2}$ is the Debye length, $L=\left(2 q T_{e} / m_{i}\right)^{1 / 2} n_{p}^{e}(0) / v_{i}$ is a characteristic length scale of the problem, $\eta=-V / T_{e}$ is the nondimensional potential, and $s=z / L, \sigma=\xi / L, l=\lambda_{c} / L$.

A formal solution of Eq. (21) is outside the scope of this paper. However, it is possible to gauge the extent of the CF-NG boundary by considering that the plasma solution [obtained by setting the left-hand side of Eq. (21) to zero] is valid over a range given by $s_{0} L$ where $s_{0}$ is a dimensionless number (independent of $L$, as long as $l$ is small [37]) on the order of $0.5[35,36]$. Assuming an average ionization rate of $5 \times 10^{15} \mathrm{~cm}^{-3} \mathrm{~s}^{-1}$ [Fig. 4(d)], we can write

$$
L=2.2 \times 10^{-10} n_{p}^{3}(0) \mathrm{mm},
$$

showing that the length of the region between the fieldreversal point and the CF is proportional to the NG density. Of more interest is the variation of the ion flux entering the $\mathrm{CF}\left(j_{i}^{\text {in }}\right)$ with the NG density:

$$
j_{i}^{\text {in }}=v_{i} s_{0} L=1.8 \times 10^{-11} n_{p}^{e}(0) s_{0} \mathrm{~mA} / \mathrm{cm}^{2} .
$$

Substituting $10^{10} \mathrm{~cm}^{-3}$ as a "typical" $n_{p}^{e}(0)$ and setting $s_{0}=0.25$ as an order-of-magnitude estimate yields $j_{i}^{\text {in }}=0.045 \mathrm{~mA} / \mathrm{cm}^{2}$, over $20 \%$ of the total discharge current (the discharge current $j$ is set at $0.2 \mathrm{~mA} / \mathrm{cm}^{2}$ in this study). While the picture is admittedly crude, we expect to see a significant ion flux entering the CF at NG densities of $10^{10} \mathrm{~cm}^{-3}$ and higher. Since the above description of the ion description function [Eq. (20)] is limited by the assumptions regarding ion-atom collisions, we treat the CF-NG region as follows.

From the extended simulation, we obtain the beamelectron behavior (ionization rate) in the discharge using the weak NG field of $10 \mathrm{~V} / \mathrm{cm}$. The CF-NG is defined as the region between the field-reversal point $\left(z=-x_{0}, E=0 \mathrm{~V} / \mathrm{cm}\right)$ and the CF $(z=0, E=10 \mathrm{~V} / \mathrm{cm})$. While it is possible in principle to use the Monte Carlo simulation to calculate the self-consistent space-charge density and electric field in the CF-NG region, we find in practice that the low-field values present numerical difficulties associated with noise in the density profiles. Therefore we use a linearly varying electric field between the field-reversal point and the CF. In contrast to the extended domain simulation in which ions created only at $z>0$ move towards the cathode, the linear field between 
$-x_{0}<z<0$ pushes the ions created there as well towards the cathode. By trial and error, we find the minimum $x_{0}$ such that the ion density resulting at the sheath edge $(z=0)$ is greater than the plasma electron density at $z=0$. The quantities adjusted at each trial value of $x_{0}$ are the potential at $z=0$, which affects the electron density through Eq. (18), and $\gamma$, which is adjusted such that the electron flux at $z=-x_{0}$ (from the extended domain simulation) is equal to the total discharge current [Eq. (7)]. It is necessary at this stage to ensure that the CF field which results is close to the one used in the extended domain simulation to generate the ionization rate.

A complete model of the CF-NG region, along the lines of solving Eq. (21), would yield a value for the space-charge density (or, equivalently, $d E / d z$ ) at the CF edge [37]. By finding the minimum $x_{0}$ that gives a positive space charge, we enforce a weaker condition that $d E / d z$ at the CF edge be positive. Further effects of the simplistic linear-field assumption are possible since the ion distribution is dependent on the field profile [e.g., Eq. (20)] and the electron density also depends on the voltage at the edge of the CF [Eq. (18)]. We test the sensitivity of our results to the assumed field by repeating the calculations using a parabolic field in the CF-NG region.

It should be noted that the minimum $x_{0}$ corresponds to the minimum ion flux $\left(j_{i}^{\text {in }}\right)$ entering the CF. We show that, counter to the argument presented in [21], this minimum ion flux is necessary at the CF edge to ensure a positive space charge at the boundary. Furthermore, we show that the amount of the ion flux which enters the CF (as opposed to being created in it) grows with the plasma electron density in the NG, as suggested by Eq. (23).

\section{EFFECT OF NEGATIVE GLOW ELECTRONS}

A sampling of the results we obtain is presented in Fig. 11 , for an assumed plasma electron density of $5 \times 10^{10}$ $\mathrm{cm}^{-3}$. We find for this density that the separation between the CF edge and the field reversal $x_{0}$ is $0.92 \mathrm{~mm}$.

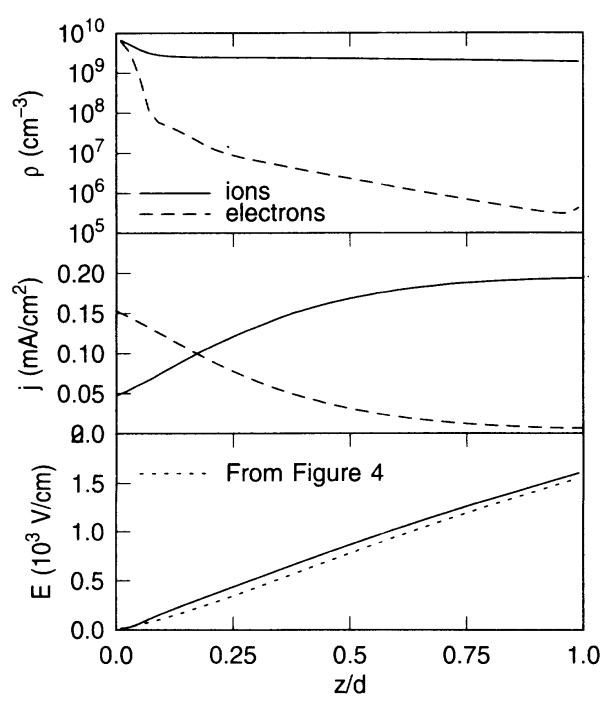

FIG. 11. Profiles obtained at plasma density of $5 \times 10^{10} \mathrm{~cm}^{-3}$.
Figure 11 shows the ion and electron densities obtained by allowing ions created up to $z=-0.92 \mathrm{~mm}$ to move towards the cathode under the assumed linear field between $z=-0.92$ and $0 \mathrm{~mm}$. The electron density shown includes the plasma electron density, attenuated by the potential at the CF edge. Therefore the numbers are much larger than the beam electron density shown in Fig. 8. Also, comparison between the ion density shown here and in Fig. 8 shows the clear need for a finite ion flux entering the CF, since the ion density must be higher than the electron density at the CF edge. Note that qualitatively the density profiles agree with those in [22], whereas those in Fig. 8 do not. Figure 11 also shows the flux of ions and electrons in the CF. The value of $j_{i}^{\text {in }}$ at this plasma density is $0.046 \mathrm{~mA} / \mathrm{cm}^{2}, 23 \%$ of the discharge current. Finally, Fig. 11 shows the electric field obtained self-consistently from the density profiles. For comparison, the field from Fig. 4 is reproduced. The change in the field appears negligible and, in fact, the CF voltage increases only by roughly $10 \%$; therefore the ionization profile from Fig. 4 used to produce the flux and density profiles in Fig. 11 is not expected to be altered by the new field.

In Fig. 12, we present the results of our parametric study of the effect of the plasma electron density. We show the variation in the length of the CF-NG boundary region and the corresponding flux of ions entering the CF. Both quantities increase monotonically with the plasma electron density, as expected from the crude model behind Eqs. (22) and (23). The trend shows that the ion flux entering the CF approaches a limiting value at higher density. The deviation from the linear behavior predicted by Eq. (23) at higher values of the plasma electron density is not surprising, considering that neither the spatial variation in the ionization rate (Fig. 4) nor the effect of elastic collisions on the ion distribution were considered in the formulation of $\mathrm{Eq} \mathrm{(23).} \mathrm{Fitting} \mathrm{a} \mathrm{line}$ through the origin and the first three data points of Fig.

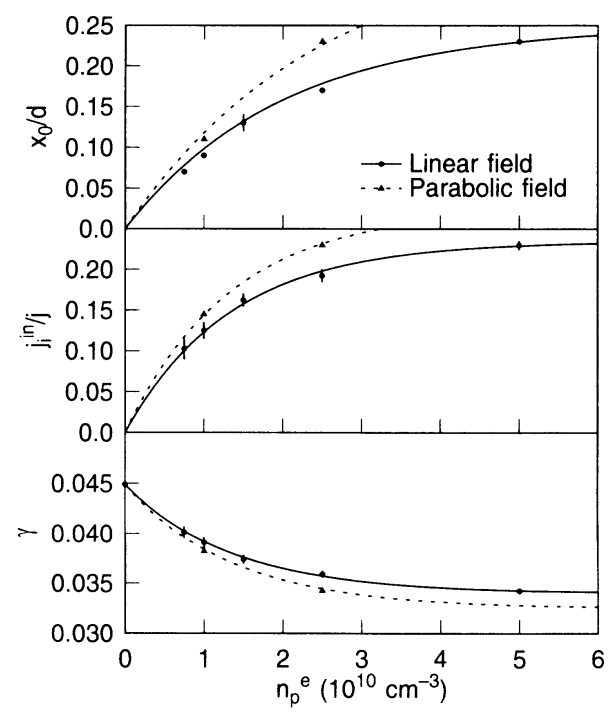

FIG. 12. Effect of plasma electron density on CF-NG boundary region. 
12(b) and comparing the slope of the fit with Eq. (23) yields a value for $s_{0}$ of 0.12 , which is not unreasonable.

Figure 12 also shows the values of $\gamma$ which correspond to the $x_{0}$ values shown in the figure. The change in $\gamma$ with the NG density is entirely an artifact of our solution scheme which keeps the sheath thickness $d$ constant. Therefore, the trend in $\gamma$ represents the tendency of the CF to shrink (if $\gamma$ were held constant instead of $d$ ) as the NG density increases.

The data shown in Fig. 12 suffer from the uncertainty of the spatial discretization (or "binning") of the Monte Carlo sampling. This uncertainty is indicated in the figure. The values of $x_{0}$ are good to within $\pm 0.04 \mathrm{~mm}$, and the corresponding uncertainty in the values of $j_{i}^{\text {in }}$ are shown with the individual points. Also shown in Fig. 12 are results (indicated by triangles) obtained at two values of the plasma electron density using a parabolic field profile in the CF-NG region. As expected, the actual values of $x_{0}$ and $j_{i}^{\text {in }}$ are sensitive to the field in the CFNG region, but the trends in the data are not. In fact, the results from the parabolic field (which is more realistic in the sense that $d E / d z$ increases along with the field towards the CF edge [37]) indicate that the true field profile may yield even larger values of $x_{0}$ and $j_{i}^{\text {in }}$ than shown in Fig. 12.

\section{SUMMARY}

Through the use of a self-consistent Monte Carlo simulation of ion and electron trajectories, we have studied the electron distribution function and the factors which affect it. The EDF near the CF-NG boundary region was shown to be affected greatly by backscatter from the NG. In spite of this coupling, it is possible to construct a separate model of the NG and apply it effectively at the edge of the CF without loss of accuracy. The use of such a model including trapped electrons in the NG, heretofore ignored, has shown that the region between the $\mathrm{CF}$ edge (defined by a threshold field value) and the fieldreversal point in the NG has an important effect on $\mathrm{CF}$ characteristics.

Specifically, we have shown with the aid of the plasma-sheath equation that a significant and critical fraction of the ion flux must enter the CF from the NG. This fraction grows with the NG density. The exact amount of the ion flux at the CF edge depends upon the gas (the collision frequencies, etc.), the conditions (discharge voltage and current), and upon the interelectrode spacing (which affects the length of the NG and its density). No attempt has been made here to study this dependence, since we have used idealized cross sections. However, it is clear from this work that ionization in the NG does contribute directly to the ion density in the CF.

\section{ACKNOWLEDGMENTS}

All computations were performed on IBM Risc System/6000 computer workstations supported by the High Performance Computing Group at Yorktown Heights. The authors wish to acknowledge the support and encouragement received from the late Dr. Ajay Sharma.
[1] B. Chapman, Glow Discharge Processes (Wiley, New York, 1980).

[2] T. J. Sommerer, W. N. G. Hitchon, and J. E. Lawler, Phys. Rev. A 39, 6356 (1989).

[3] T. J. Sommerer, J. E. Lawler, and W. N. G. Hitchon, J. Appl. Phys. 64, 1775 (1988).

[4] T. C. Paulick, J. Appl. Phys. 67, 2774 (1990).

[5] P. Bayle, J. Vacquie, and M. Bayle, Phys. Rev. A 34, 360 (1986); 34, 372 (1986).

[6] J. H. Ingold, in Gaseous Electronics, edited by M. N. Hirsch and H. J. Oskam (Academic, New York, 1978), Vol. 1., p. 24.

[7] K. G. Muller, Z. Phys. 169, 432 (1962).

[8] L. Friedland, J. Phys. D 7, 2246 (1974).

[9] J. P. Boeuf and E. Marode, J. Phys. D 15, 2169 (1982).

[10] M. Ohuchi and T. Kubota, J. Phys. D 16, 1705 (1983).

[11] N. Sato and H. Tagashira, J. Phys. D 18, 2451 (1985).

[12] T. J. Moratz, L. C. Pitchford, and J. N. Bardsley, J. Appl. Phys. 61, 2146 (1987).

[13] T. J. Moratz, J. Appl. Phys. 63, 2558 (1988).

[14] T. Itoh and T. Musha, J. Jpn. Phys. Soc. 15, 1675 (1960).

[15] R. W. L. Thomas and W. R. L. Thomas, J. Phys. B 2, 562 (1969).

[16] Y. Sakai, H. Tagashira, and S. Sakamoto, J. Phys. B 5, 1010 (1972).

[17] D. Bhasavanich and A. B. Parker, Proc. R. Soc. London, Ser. A 358, 385 (1977).
[18] A. V. Phelps and L. C. Pitchford, Phys. Rev. A 31, 2932 (1985).

[19] A. V. Phelps, B. M. Jelenkovic, and L. C. Pitchford, Phys. Rev. A 36, 5327 (1987).

[20] N. A. Tran, E. Marode, and P. C. Johnson, J. Phys. D 10, 2317 (1977).

[21] E. A. Den Hartog, D. A. Doughty, and J. E. Lawler, Phys. Rev. A 38, 2471 (1988).

[22] M. Surendra, D. B. Graves, and G. M. Jellum, Phys. Rev. A 41, 1112 (1990).

[23] W. P. Allis, in Elektronen Emission Gasentladungen I, edited by S. Flügge, Handbuch der Physik Vol 21 (Springer, Berlin, 1956), p. 383.

[24] M. Hayashi, Institute of Plasma Physics (Nagoya University) Report No. IPPJ-AM-19, 1981 (unpublished).

[25] E. Eggarter, J. Chem. Phys. 62, 833 (1975).

[26] L. R. Peterson and J. E. Allen, Jr., J. Chem. Phys. 56, 6068 (1975).

[27] M. L. Vestal, C. R. Blakley, and J. H. Futrell, Phys. Rev. A 17, 1337 (1978).

[28] D. A. Doughty, E. A. Den Hartog, and J. E. Lawler, Phys. Rev. Lett. 58, 2668 (1987).

[29] R. T. Farouki, S. Hamaguchi, and M. Dalvie, Phys. Rev. A 44, 2664 (1991).

[30] M. Dalvie, R. T. Farouki, S. Hamaguchi, and M. Surendra, IBM Research Report No. RC 17723, 1992 (unpublished). 
[31] J. E. Lawler, Phys. Rev. A 32, 2977 (1985).

[32] L. S. Frost, Phys. Rev. 105, 354 (1957).

[33] P. Gill and C. E. Webb, J. Phys. D 10, 299 (1977).

[34] K. G. Emeleus, J. Phys. D 14, 2179 (1981).

[35] L. Tonks and I. Langmuir, Phys. Rev. 34, 876 (1929).

[36] E. R. Harrison and W. B. Thompson, Proc. Phys. Soc. 74,
145 (1959).

[37] S. A. Self, Phys. Fluids 6, 1762 (1963).

[38] J. T. Scheuer and G. A. Emmert, Phys. Fluids 31, 3645 (1988).

[39] W. D. Davis and T. A. Vanderslice, Phys. Rev. 131, 219 (1963). 


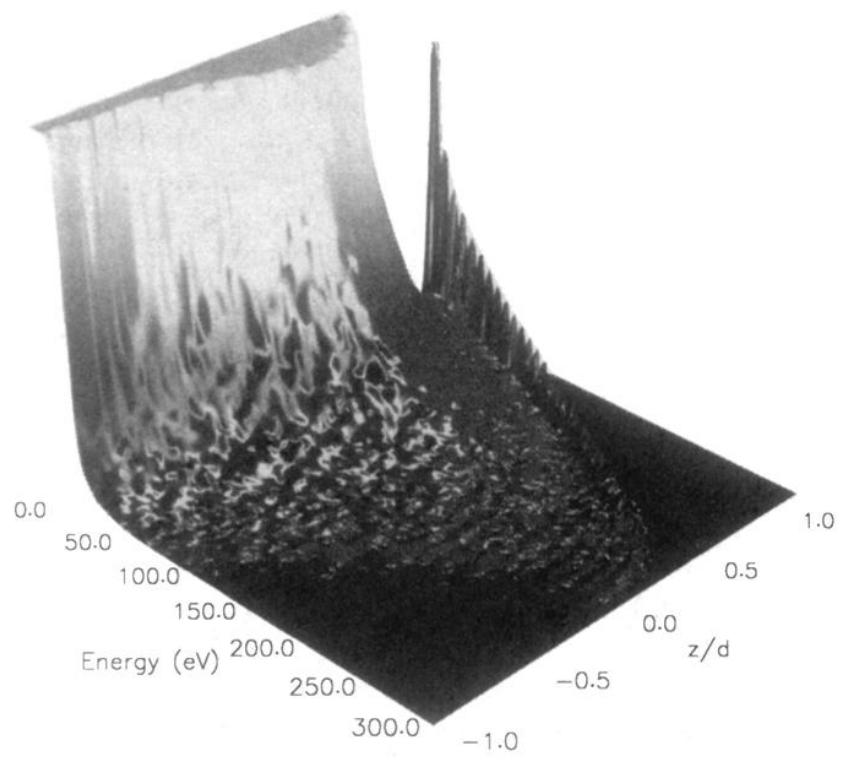

FIG. 6. EEDF as a function of position. 